\title{
Some considerations for precision metrology of thin $x$-ray mirrors
}

\author{
J. P. Lehan ${ }^{\mathrm{a}, \mathrm{b}}$, T. Saha ${ }^{\mathrm{b}}$, W. W. Zhang ${ }^{\mathrm{b}}$, S. Rohrbach ${ }^{\mathrm{b}}$, K.-W. Chan ${ }^{\mathrm{a}, \mathrm{b}}$, T. Hadjimichael ${ }^{\mathrm{b}, \mathrm{c}}$, M. Hong ${ }^{\mathrm{b}, \mathrm{d}}$, \\ and W. Davis $\mathrm{e}^{\mathrm{e}}$ \\ ${ }^{a}$ Center for Research and Exploration in Space Science and Technology, NASA Goddard Space \\ Flight Center, and Department of Physics, University of Maryland, Baltimore County, \\ 1000 Hilltop Circle, Baltimore, Maryland 21250 \\ ${ }^{\mathrm{b}}$ NASA Goddard Space Flight Center, Greenbelt, Maryland 20771 \\ ${ }^{c}$ Ball Aerospace, 1616 McCormick Drive, Upper Marlboro, MD 20774 \\ ${ }^{\mathrm{d} S G T}$, Inc., 7701 Greenbelt Road Suite 400, Greenbelt, Maryland 20770 \\ ${ }^{\mathrm{e}}$ Harvard-Smithsonian Center for Astrophysics, 60 Garden Street, Cambridge, MA 02138
}

\begin{abstract}
Determination of the shape of very thin x-ray mirrors employed in spaced-based telescopes continues to be challenging. The mirrorsôshapes are not readily deduced to the required accuracy because the mount induced distortions are often larger than the uncertainty tolerable for the mission metrology. In addition to static deformations, dynamic and thermal considerations are exacerbated for this class of mirrors. We report on the performance of one temporary mounting scheme for the thin glass mirrors for the Constellation-X mission and prospects for deducing their undistorted shapes.
\end{abstract}

Keywords: $\mathrm{x}$-ray telescopes, Constellation-X, mirror mounting, optomechanics

\section{INTRODUCTION}

$\mathrm{X}$-ray astronomy is limited to space-based observations. Traditionally this, in turn puts severe strain on either the entendu of the telescope (Chandra) or the resolution (Suzaku). In fact, neither trade is desirable from the point of view of the x-ray astronomer. On the other hand, many compact objects such as the event horizons of black holes are too small to be imaged by even the best modern telescopes. Given these objects cannot be resolved, the resolution needed by an x-ray telescope then can be rather modest by optical telescope standards for resolving other point sources from one another.

The grazing-incidence nature of x-ray telescopes greatly reduces their collecting area. As a result, an x-ray telescope is in fact a number of nested, confocal telescopes. The number of confocal elements and their individual areas sets the collection area. To make this as large as possible and still be within the payload limits of the launch vehicle, most $\mathrm{x}$-ray telescopes consist of very thin shells. This comes with a penalty that their fabrication is difficult and often imprecise and they are easily distorted when mounted. This second penalty can away in theory while orbiting but since gravity distortion is present during terrestrial assembly, usually not completely.

Additionally, the component fabrication process is uncertain because of the distortions present during metrology either results in incorrect processing (surface correction) or unacceptably large uncertainty in the quality at the component level. It is this metrology mounting problem that we address here.

There are two basic approaches to the mirror mounting problem: 1) Make a mount that distorts the mirror much less than the metrology requirements for the mirror, or 2) Make a mount whose distortions are repeatable and predictable to sufficient degree that they can be removed from the measurement. We have opted to pursue the second approach although the distortions cannot be too large for them to be repeatable. 
A metrology mount can be either a temporary mount or permanent mount. A permanent metrology mount becomes part of the final telescope assembly. If temporary, ideally it should have no residual footprint when the mirror is mounted in the permanent flight telescope structure. That is, it should not leave any trace of its presence when the mirror is mounted in the final assembly. It is advantageous if the metrology mount also serves as a transfer mechanism into the permanent housing. We discuss here only temporary mounting concepts although one could conceive of ways they might be modified to serve as permanent mounts.

\section{MIRROR MOUNT KINEMATICS}

\subsection{Statics: Self-weight distortion and gravity orientation}

For the Constellation-X telescope, the baseline mirror is well approximated for mechanical purposes as an azimuthal section of a cone $200 \mathrm{~mm}$ in axial extent and only 400 microns thick. Thus, in any mount, its self-weight distortion is significant. (By significant, we mean the self-weight distortion exceeds the metrology tolerances.) The distortion, however, reaches a minimum near the orientation of the gravity vector being parallel to the cone axis $\ddot{i}$ but not exactly at that point. Since we are dealing with only a section of a cone and not the entire cone, it turns out that there is an angle slightly away from vertical that yields a minimum in distortion. The exact angle depends upon the cone half angle and the azimuthal extent of the cone. This angle may be thought of as the angle with the minimum average angular deviation of the surfaces from vertical. For example, for a cylinder, this angle is exactly vertical resulting in no component of the gravity vector normal to the cylinder surface. For a cone, there is no angle that meets the equivalent condition so it becomes a minimization problem. Figure 1 shows the P-V distortion of a particular mirror prescription as a function of angle. Note that the minimum is rather broad indicating a weak dependence on the distortion with angle near the minimum. This reduced sensitivity makes mounting in this way practical.

If the gravity vector is nearly normal to the surface (say, normal to the centerline of the mirror segment) the self-weight distortions will, in general, reach a maximum. For a simply-supported kinematic-type mount, the self-weight distortions are many times the metrology accuracy required for the mission. Previously, we employed a non-kinematic, selfadjusting low distortion mount ${ }^{1}$ with many soft springs to counteract the gravity self-weight distortions in this nearsurface-normal gravity orientation. Experience with the mount has concluded, however, that the mountôs selfadjustment mechanism was very sensitive to initial conditions (mirror placement) and so we have adopted new approaches in the near-vertical orientation which we discussed here.

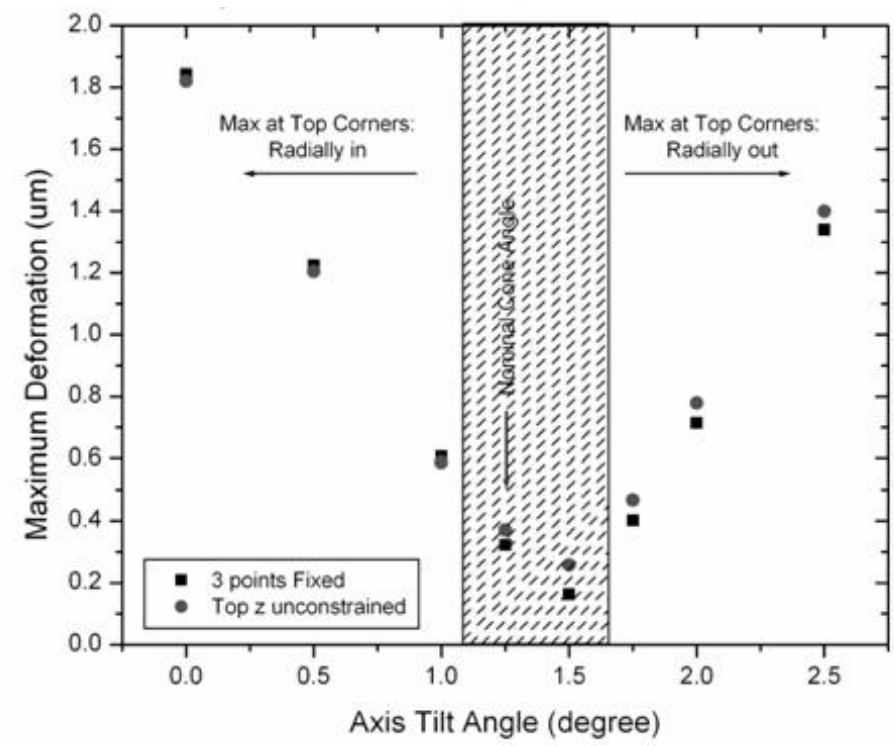

Fig. 1. The peak-to-valley deformation of a Constellation-X mirror of $60^{\circ}$ azimuthal extent in a simple 3-pt kinematic mount. In this case the cone opens down and the cone half-angle is indicated in the figure. 


\subsection{Dynamics}

The thin nature of the Constellation-X mirrors result in significant dynamic surface displacements resulting from ambient vibration sources (acoustic and seismic). Thus, in addition to the static, self-weight gravity distortion, the shape is time dependent. As a result, only the time averaged shape will resemble the expected self-weight distortion. It behooves us to also consider the dynamic behavior of the mount-part combination when designing a mount. Figure 2 shows two extreme shapes of the fundamental mode of a Constellation-X mirror in a three-point kinematic mount.
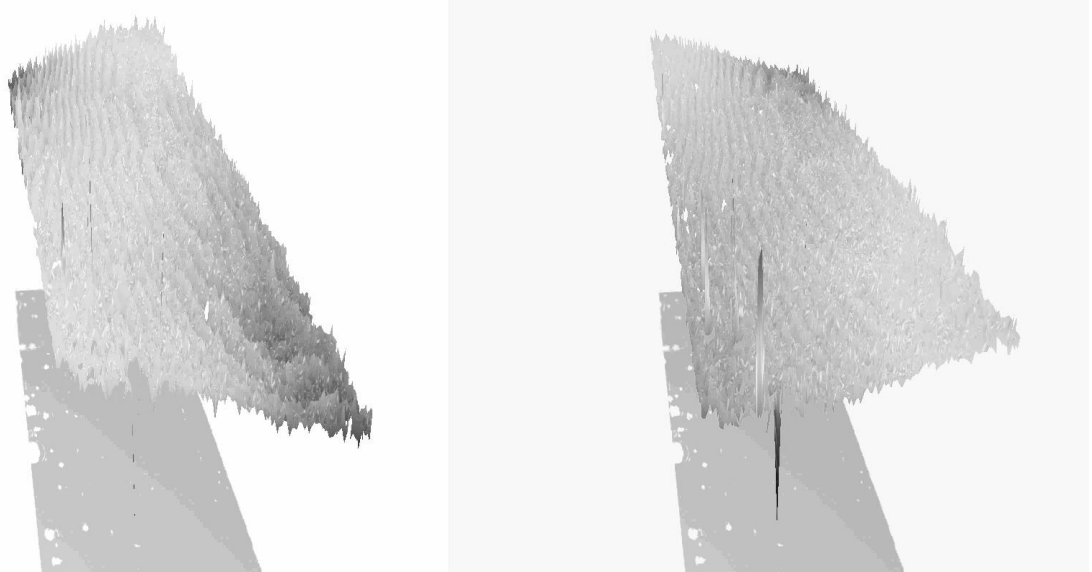

Fig. 2. The measured extreme shapes of the fundamental mode of vibration for a $30^{\circ}$ azimuthal extent Constellation-X mirror in a simple 3-pt kinematic mount captured with the FizCam 1500. Data is difference from the average of 100 measurements.

In dealing with the part-mount dynamics, we also need to take into account the nature of the measurement. Ideally, the mode frequency and mode shape should be tailored to have a minimum impact on the metrology being performed. In the case of vibrational frequency, the practical target is set by the capture rate of the interferometer employed. Our 4D Technologies FizCam ${ }^{\circledR} 1500$ interferometer frame rate is limited to 14 frames per second so the maximum frequency that can be sampled at Nyquist is $7 \mathrm{~Hz}$. Figure 3 shows the predicted frequency spectrum in one of our mounts. This indicates we are undersampled. Things are not quite as bad as it might seem because the environmental sources of vibration are random in amplitude, frequency content, and time (phase). Thus, by averaging a large number of samples (50 in practice) we can beat down the metrology errors resultant from vibration.

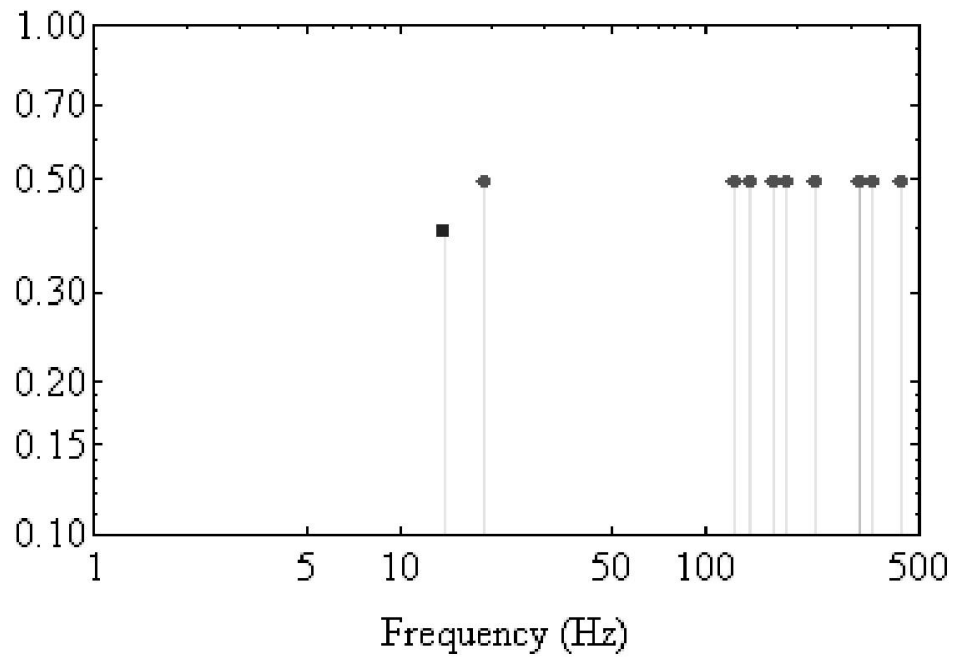

Fig. 3. The calculated frequency spectrum of a $30^{\circ}$ azimuthal extent Constellation-X mirror and metrology mount combination (circles). The maximum sampling rate of the FizCam 1500 is also indicated by the square. 
The mode shape is also important. For mirror mode shape, we have been given a small reprieve in the requirements by the nature of the problem. For the grazing-incidence Wolter Type I telescopes we employ, the performance is only about a tenth as sensitive to azimuthal errors as axial errors. This provides some design room for the metrology mount. If one can shape the modes such that the time derivative of the axial slope approaches zero, then the axial metrology will have a minimum error. Stated another way, if the modes shapes are separable into axial and azimuthal components, and the time-dependant part is purely azimuthal, then the vibration will have no influence on the axial component of the metrology.

\section{NEAR-VERTICAL MOUNTING CONCEPTS}

We have taken two approaches for the temporary mount. Both operate in the near-vertical gravity orientation. The first is based on a fixed-support non-kinematic mounting scheme. The steps involved are as follows: The mirror is hung by two fine threads or wires located on the perimeter of the mirror in the same plane as its center of mass $(\mathrm{CM})$. The mirror is bonded at four points near the Airy points on its back. Conceptually, the threads are much more compliant than the mirror so they introduce a minimum of distortion. By hanging in the plane of the CM, the mirror is not twisted. In addition, the long threads allow a minimum of normal force on the mirror when put in contact with the four bonding points. This mounting scheme has been dubbed the ñSuspensionò Mount and is discussed in greater detail in Chan, et $\mathrm{al}^{2}$ in these proceedings.

The second method is a kinematic temporary mount. We have mentioned this mount previously ${ }^{3}$ and dubbed it the ñCantor Treeòmount because its whifflingố passing resemblance to the Cantor set. Here we will further elucidate the properties and design considerations involved in its construction. Unlike the Suspension Mount, in the Cantor Tree mount the mirror is placed in the mount and the mirror angle relative to gravity adjusted as desired. For most of the results presented here, that is such that the centerline of the mirror is vertical. This angle was chosen primarily out of convenience because it is readily found to a few arc-sec accuracy with little difficulty and is the same for all mirror prescriptions. We will be employing the minimum-distortion angle mentioned above (see also Fig. 1) in the near future.

The mount is shown in Figure 4 with a mirror mounted. The inset shows a close-up of a portion of the bottom interface between the mount and the mirror itself. The mount consists of a cascade of pivots. Working from the mirror outward on the bottom, we have a slot into which the mirror slides that pivots (roughly) in the plane defined by the surface normal and mirror tangent. The mount accommodates a wide range of local edge slopes of the mirror without introducing a measurable local distortion. This pivot is primarily to allow a range of mirrors sizes in the telescope to have a common mount for metrology. For example, all of the mirrors for the Optical Alignment Pathfinder ${ }^{4}$ can be accommodated with the mount pictured in Fig. 4. The next level of pivots rotates in a plane parallel to that of the slots and pivots like a seesaw in a plane perpendicular to it. This see-saw rotation allows each point to bear (approximately) equal loads so that the distortion at each load point is a minimum. The attachment is then to a solid mount that can be manipulated kinematically, if desired. We employ four attachment points on the bottom for mirrors between 40 and 60 degree angular span and two points for mirrors of smaller angular span. Figure 4 also shows the upper half of the mount. In this case there is an additional splitting (whiffling) from the part interface to the hard mounting so that it reduces to a single kinematic attachment point for manipulation. Thus, in total, the mount reduces to a three-point kinematic mount for rigid-body motions. All the motions are shown schematically in Figure 5 for the case of four lower and four upper mirror contact points.

For a static load, the upper branch constrains the mirror only radially (normal to the mirror surface). The distortion introduced, however, will depend upon the angle at which the mirror is held relative to the gravity vector and the cone angle of the mirror (mirror prescription). The proper selection of angle can result in a very low distortion. Figure 6 shows the displacements for the case of the centerline vertical. The distortion for this case represents a 0.98 arc-sec RMS error in the shape of the mirror induced by the mount.

The primary function of the upper ñoranchò of the Cantor Tree mount, however, is to modify the shape and amplitude of the vibrational modes. We have chosen the mirror attachment points so that the mode shapes are as close to purely azimuthal as practical to minimize the influence on the metrology as discussed above. This is accomplished by having 
the upper ñaranchesò of the Cantor Tree mirror the lower branches (see Figure 4). The second mode shown in Figure 7 is almost purely azimuthal in character, as desired..

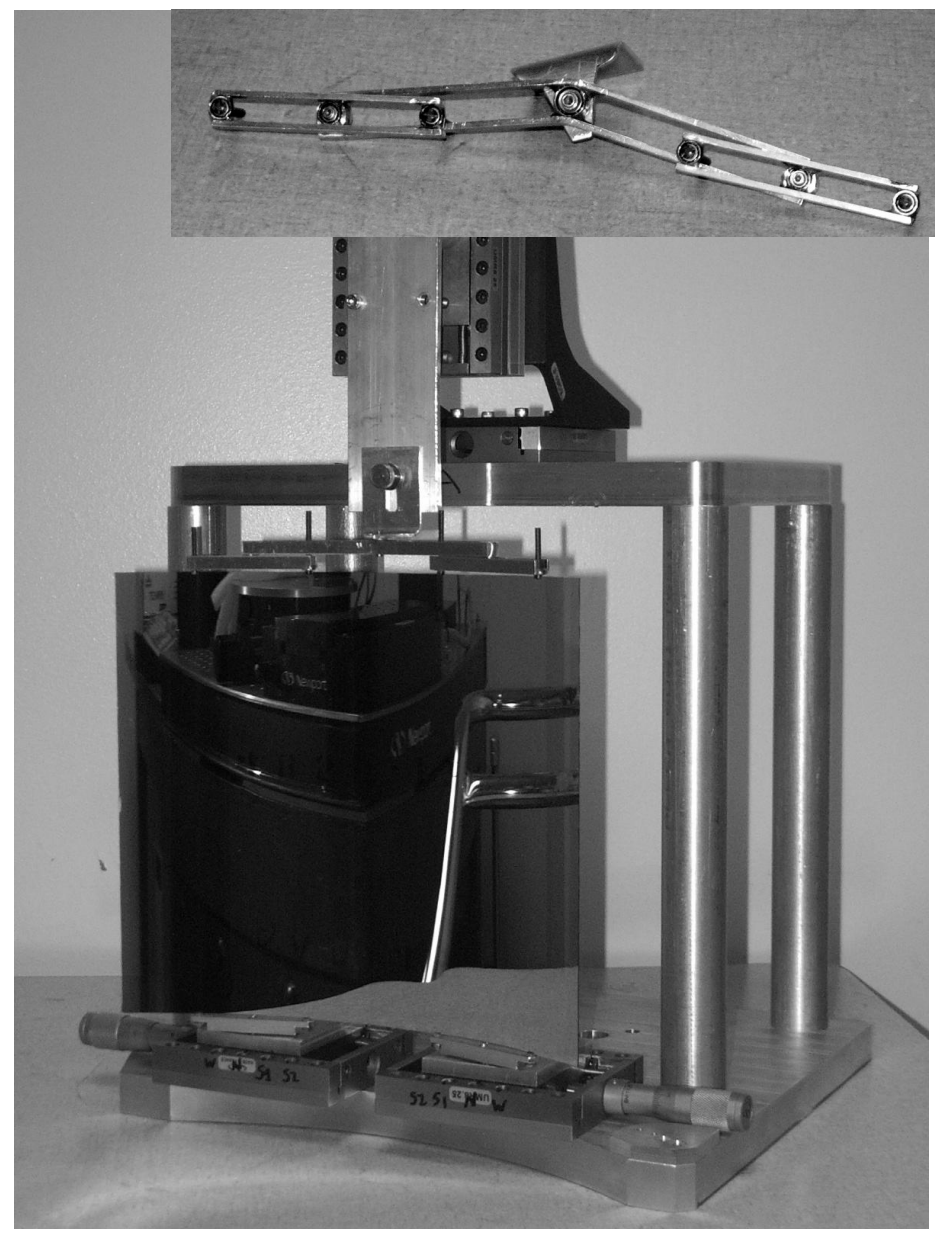

Fig. 4. The ñCantor Treeò mount for mirrors ranging from $40^{\circ}$ to $60^{\circ}$ azimuthal extent with adjustable bottom branches. Inset: Upper branch showing detail of pivots.

Fig. 5. The ñCantor Treeò Mount of Fig. 4 for mirrors ranging from $40^{\circ}$ to $60^{\circ}$ azimuthal extent illustrating the degrees of freedom of the mount [after Lehan, et $\mathrm{al}^{6}$ ]. The bottom branches are directly analogous to the more-familiar whiffle tree mount. The whiffling of the upper branch is primarily for vibrational mode shaping although it also constrains the mirror radially (normal to the mirror surface).

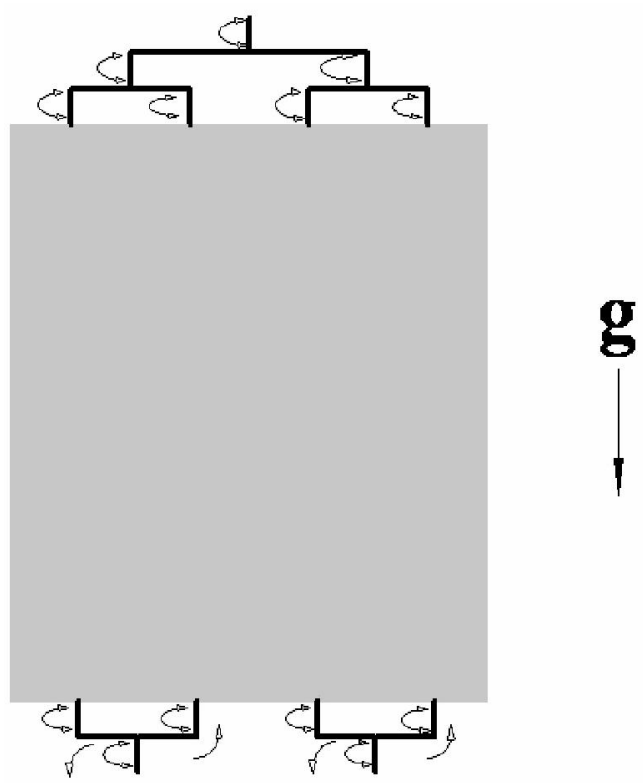



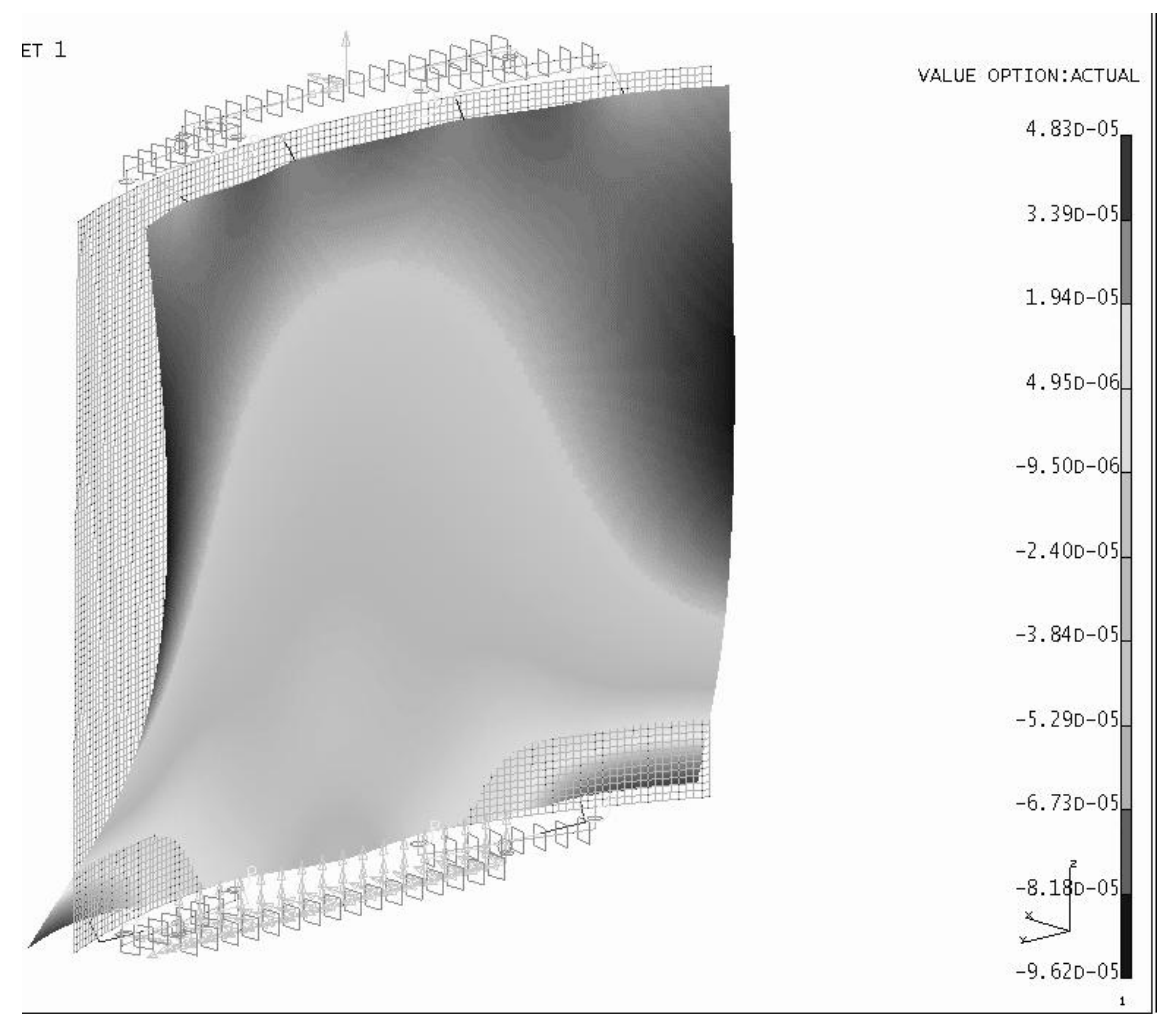

Fig. 6. Calculated displacements of a Constellation-X primary mirror in the Cantor Tree mount of Figure 4 for the centerline vertical case. Displacements are in $\mathrm{mm}$.

The primary function of the upper ñbranchò of the Cantor Tree mount, however, is to modify the shape and amplitude of the vibrational modes. We have chosen the mirror attachment points so that the mode shapes are as close to purely azimuthal as practical to minimize the influence on the metrology as discussed above. This is accomplished by having the upper ñoranchesò of the Cantor Tree mirror the lower branches (see Figure 4). The second mode shown in Figure 7 is almost purely azimuthal in character, as desired.

The mode frequency and amplitude can be tuned somewhat by varying the masses of the branches. For metrology, our goal is not the same as it would be for the launch payload. For launch it is desirable to have as high a fundamental frequency and as low an amplitude as possible without sharp resonances. For metrology, a low amplitude is also desirable but we want a low fundamental frequency. The reason for the low frequency is that this puts us in the capture range of the 4D FizCam 1500 interferometer. This is accomplished by increasing the mass of the branches. There is a limit to the extent in which this methodology can be applied because the mass of a branch must be less than the mirror as a whole or the mirror will vibrate independently of the mount. If the fundamental mode frequency cannot be brought into the capture range of the interferometer, then the amplitude must be $<<1$ fringe or $<60 \mathrm{~nm}$. Obviously, there are a plethora of other Cantor Tree configurations that would yield other mode shapes and frequency spectra so that one or the other criterion required for accurate metrology can usually be met.

\section{RESULTS}

Comparison between the finite element models and measurements is complicated by the fact that the undistorted shape of the mirrors prior to mounting is not known. Instead we use as a measure of comparison the mandrel shape which is the shape targeted during the mirror forming process. (That is, a flawless forming will exactly replicate the mandrel.) Further complicating the interpretation of the results is the present state of our metrology. We are currently limited to a

36 degree field of view of the mirrors by the design of the cylindrical null lens.5 Although we can stitch together overlapping 36 degree views, there is some ambiguity in the resultant overall shape.6 This ambiguity affects the piston 


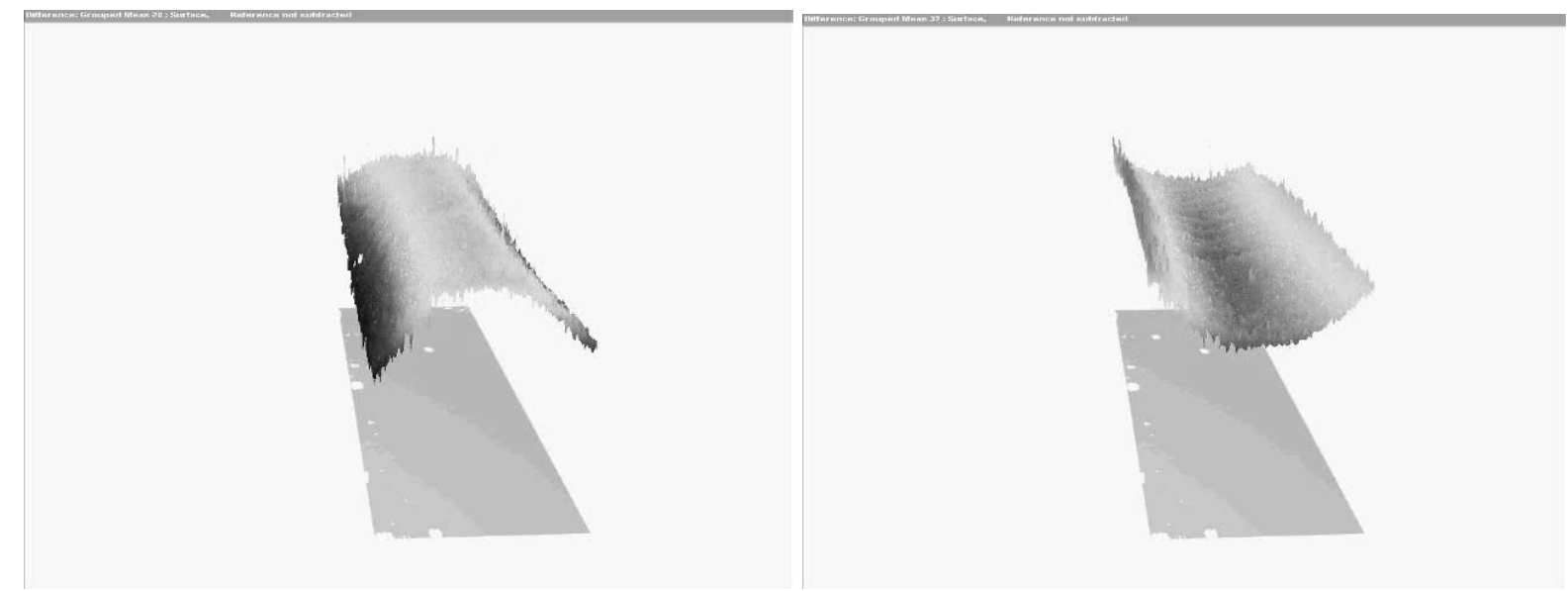

Fig. 7. Extreme shape for mode 2 oscillation of a $30^{\circ}$ azimuthal extent mirror in a Cantor Tree mount. Note that it is almost completely azimtuhal in character.

and tilts as a function of azimuth on the mirror [op in ASCA terminology7]. Higher orders are not affected. We examine only these higher order errors in Fig. 8(a). To aid the interpretation, the mandrel form is subtracted from the results. We see that the distortions are much worse at the azimuthal edges of the part than what is expected from the finite element prediction [Fig. 8(b)] of the mirror distortions. Over the majority of the mirror, however, the distortions are small, in agreement with the modeling. This suggests the edges of the mirror are distorted by processes other than mounting distortion. We are currently trying to identify the causes of this edge distortion.

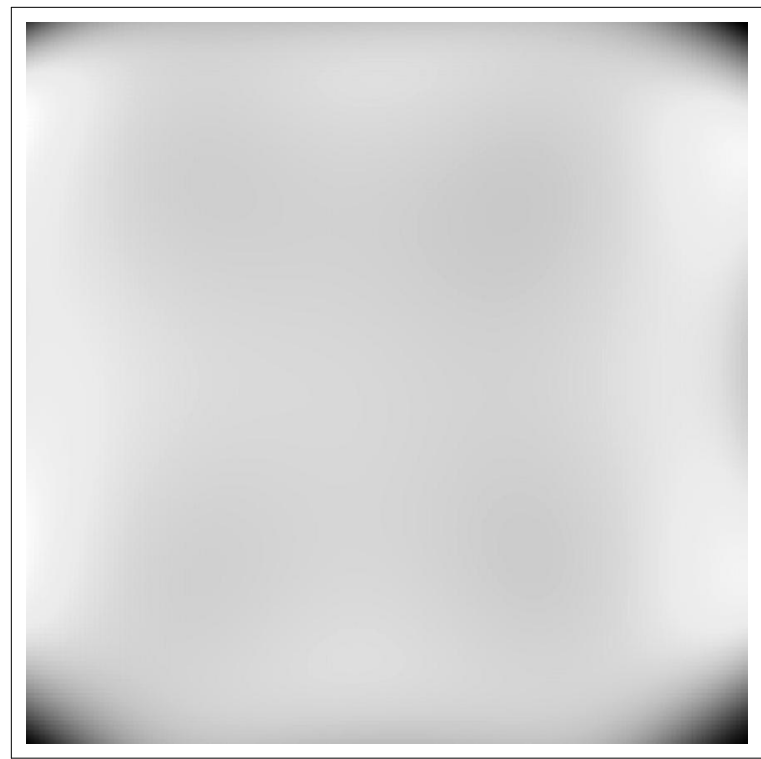

(a)

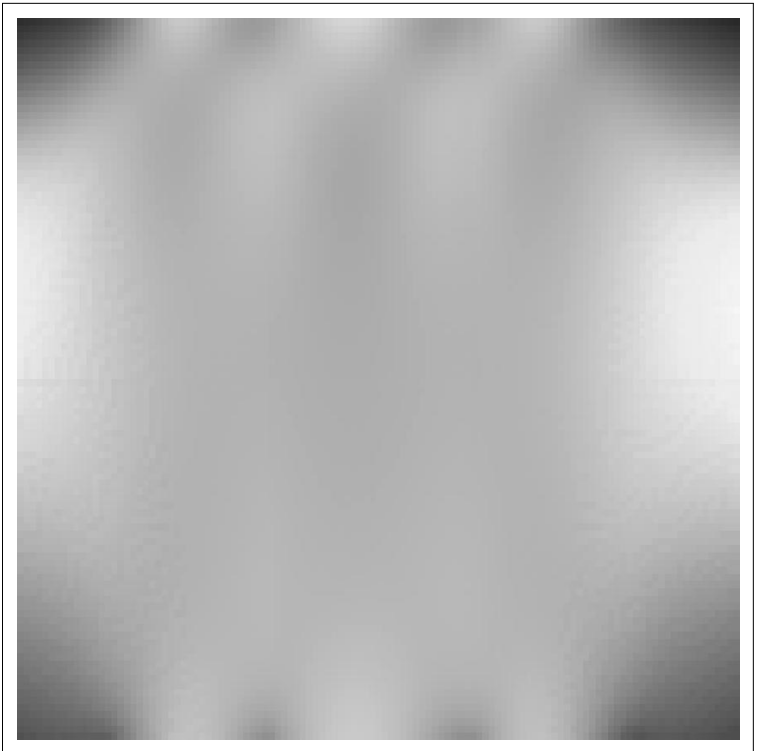

(b)

Fig. 8. (a) Measured and (b) calculated higher-order distortions $\left(2^{\text {nd }}-6^{\text {th }}\right.$ order) of a Constellation-X primary mirror in the Cantor Tree mount. The scale in (a) is -2.5 to 0.7 micron whereas the scale in (b) is -0.2 to 0.1 micron. The axial direction is vertical in the figures. Note: (b) corresponds to Fig. 6 with cone angle and grop removed. (See text for further discussion.)

\section{SUMMARY}

We discuss some considerations involved in mounting thin mirrors for metrology, These are static and dynamic consisderations. Unlike the more familiar behavior of thick terrestrial mirrors or even lightweighted space optics, the 
thin x-ray mirrors show significant vibration even under normal optical metrology laboratory conditions. This vibration then needs to be considered and planned for when mounting and measuring the mirrors. The static self-weight distortions can also be very large and so the mounts need to deal with that as well. The self-weight distortions are a minimum near vertical for this type of mirror so we discuss mounts taking advantage of this fact. We discussed the Cantor Tree mount which is inspired by the whiffle tree mount employed in mounting large terrestrial telescope mirrors but adapted to the particulars of x-ray mirror metrology.

\section{OTHER SOLUTIONS}

We do not pretend that our chosen solutions are the only- possible solutions and we mention a few possibilities in passing here. One appealing idea is a ñzero gravityò mount realizable by suspending the mount in a density-matched solution. A solution of polytungstate is a good non-carcinogenic possibility commonly used to determine the density of minerals or for mineral separation. Polytungstate solutions are very clear at the $632.8 \mathrm{~nm}$ line of Ne employed in the majority of commercial interferometers and can be made in very high concentrations of solute-to-water. Above a certain point, however, the solution properties become very sensitive to concentration variations. In addition, like many salts, it reduces the effective cohesive (polar) forces of the solvent (water) and thus produces a humid environment above the solution. This further concentrates the solution with a concomitant alteration of the solution density and refractive index. Thus, the air volume above the solution needs to be minimized and enclosed. In addition, we found that at the high concentrations needed to match the density of the glass $(2.54 \mathrm{~g} / \mathrm{cc})$, the solutions formed a small-but-apparent increasing density gradient in the solution from top to bottom (and an concomitant refractive index gradient). This could be eliminated by stirring but stirring was not compatible with our optical measurements. Thus, we abandoned this method although it probably would be compatible with a non-optical metrology technique.

An intriguing mount configuration is a four point mount (4 planar points) with perfect inversion. If a part is simply supported at four points with the gravity vector normal to the midpoint of the mirror and then perfectly inverted and remeasured, the average of the two measurements is the undistorted mirror shape. The real practical advantage of this method is that it does not rely on the properties of the mirror material being known, or the even the same from point-topoint on the mirror. The error is the imperfection of the inversion. This measurement will be attempted when a full aperture null lens becomes available in the near future.

\section{REFERENCES}

${ }^{1}$ William W. Zhang, Kai-Wing Chan, John P. Lehan, and Robert Petre, r̃Fabricate and assemble: an alignment and integration method for next generation x-ray telescopes, ò Proc. SPIE 6266, 62661M (2006).

${ }^{2}$ Chan, et al, these proceedings

${ }^{3}$ Lehan, J. P., S. Owens and T. Hadjimichael, ñToward a complete metrologic solution for the mirrors fro the Constellation-X spectroscopy x-ray telescope,ò Proc. SPIE 6688 668818-1-18 (2007).

${ }^{4}$ Scott M. Owens, Jason H. Hair, Jeffrey W. Stewart, Robert Petre, William W. Zhang, William A. Podgorski, Paul E. Glenn, David A. Content, Timo T. Saha, and Giriraj Nanan, ñConstellation-X SXT optical alignment Pathfinder 2: design, implementation, and alignment,ò Proc. SPIE 5168, 239 (2004).

${ }^{5}$ J. P. Lehan, T. Hadjimichael, D. A. Content, and W. W. Zhang, ñDesign and fabrication of refractive nulls for testing the segmented mirrors of the Constellation-X spectroscopy x-ray telescope (SXT), ò Proc. SPIE 5900, 59001D (2005). ${ }^{6}$ J. P. Lehan, T. Hadjimichael, and C. Skocik, ñTesting of the mirrors for the Constellation-X spectroscopy X-ray telescope with a refractive null,ò Proc. SPIE 6688, 668819 (2007).

${ }^{7} \mathrm{P}$. Glenn, "Set of orthonormal surface error descriptors for near-cylindrical optics", Opt. Eng., 23 384-390 (1984). 